\section{FUENTES DE LA INNOVACIÓN Y EL PAPEL DE LAS INSTITUCIONES EN EL SISTEMA DE INNOVACIÓN TEXTIL DE UN DISTRITO INDUSTRIAL VALENCIANO}

\author{
Emilio Golf-Laville \\ Departamento de Organización de Empresas \\ Universidad Politécnica de Valencia \\ emgolla@omp.upv.es \\ Francisco Javier Ortega-Colomer \\ Instituto de Gestión de la Innovación y el Conocimiento (INGENIO) \\ CSIC-UPV \\ fraorco@ingenio.upv.es
}

\begin{abstract}
In this article we analyse the shift that the textileclothing sector has recently suffered in the Valencian Central Counties from the role played by the institutions of the local innovation system. This paper includes the main results derived from different research projects undertaken between 2004 and 2009. The analytical framework used is based on the innovation systems approach, which emphasizes not only the existence of organizations supporting the industry, but also their behavioural relationships. Thus, we identify the sources of innovation in an industry localized in a peripheral region with low technological intensity. This sector currently presents a structural adjustment process derived from the opening of international markets.
\end{abstract}

KEY WORDS: Low- and medium-technology industries; industrial districts; sources of innovation; businesses strategies; peripheral regions.

\section{INTRODUCCIÓN}

En ocasiones, los datos referentes a actividades de innovación a nivel local (tanto de inputs como de outputs) no se encuentran disponibles en ninguna base de datos oficial al uso. A esto hay que añadir que los indicadores cuantitativos disponibles no suelen reflejar todos los aspectos que son relevantes para explicar fenómenos complejos tales como los procesos de innovación. Este hecho impulsó a un grupo de investigadores a estudiar en profundidad, durante ya una década, un sector productivo en particular, el textil-

\author{
INNOVATION AND \\ INSTITUTIONS: A CASE STUDY \\ OF A TEXTILE INNOVATION \\ SYSTEM IN A VALENCIAN \\ INDUSTRIAL DISTRICT
}

RESUMEN: El artículo analiza el cambio en la industria textil-confección de las Comarcas Centrales Valencianas (CCV, en adelante) a partir del papel desempeñado por las instituciones que conforman el sistema local de innovación. Este trabajo recoge los principales resultados derivados de diferentes proyectos de investigación realizados entre los años 2004 y 2009. El marco analítico empleado se sustenta en el enfoque de sistema de innovación, que hace especial énfasis tanto en la existencia de instituciones de soporte a la industria, como en sus relaciones de comportamiento. De este modo, se identifican las fuentes de innovación de una industria localizada en una región periférica, con baja intensidad tecnológica, que en los últimos años presenta un proceso de ajuste estructural derivado de la apertura internacional de mercados.

PALABRAS CLAVE: Industrias de baja y media tecnología; distritos industriales; fuentes de innovación; estrategias empresariales; regiones periféricas.

confección, localizado en un distrito industrial específico, concretamente en las CCV (Golf-Laville et al., 2005; Alto Consejo Consultivo, 2007; Golf-Laville et al., 2008). Con estos estudios se realizó una aproximación al papel de la innovación en los procesos de cambio empresarial y sectorial, objeto de un interés creciente tanto a nivel académico como político, atendiendo a las particularidades de una región funcional caracterizada por mantener una posición periférica y un desarrollo económico históricamente basado en la preeminencia de una industria de baja intensidad tecnológica. 
Actualmente, al igual que en otras áreas productoras tradicionales, la industria textil de esta zona está afrontando diferentes retos con respecto a tres áreas clave: mercados, tecnologías y productos. En primer lugar, los mercados están cambiando a causa de la creciente competitividad, fruto de la liberalización internacional. En segundo lugar, la industria textil ha sido caracterizada por su dependencia en el desarrollo de tecnología en otros sectores, como la construcción de maquinaria, la industria química o las tecnologías de la información y la comunicación. Y en tercer lugar, la presión hacia la diferenciación del producto, como estrategia alternativa a la reducción de costes, ha conducido a una continua y creciente diversificación de la oferta de productos y servicios. En este contexto, la necesidad de innovar se ha incrementado enormemente, no sólo desde el enfoque tradicional de productos y procesos, sino principalmente desde el punto de vista organizativo (cadena de suministro, outsourcing, etc.) y de negocio (e-business, franquicias, etc.), generando una nueva configuración de las relaciones que empresas y agentes locales desempeñaban en este tipo de aglomeraciones industriales. De hecho, la complejidad y profundidad de este proceso de cambio, requiere de una aproximación al comportamiento de estas aglomeraciones, más allá de los tradicionales enfoques del distrito industrial marshalliano, en los que el resultado innovador y productivo dependia, principalmente, del conocimiento localizado. Este artículo provee precisamente de un análisis exhaustivo de los principales agentes que componen la industria textil utilizando el enfoque de sistemas de innovación $y$, por tanto, incluye, además, el papel de los factores externos al territorio.

Este trabajo resume los resultados derivados de diferentes proyectos en los cuales los investigadores han participado en los últimos años. El artículo parte de una primera sección en la cual se revisa el marco teórico (sección 2), para, a continuación, realizar una breve exposición de las fuentes de información empleadas (sección 3). La tercera sección caracteriza tanto a la industria (sección 4), como al territorio objeto de estudio por separado (sección 5), para luego discutir el papel y las interrelaciones de los principales agentes que conforman el sistema de innovación textil en esta zona (sección 6). Por último, se discuten los principales resultados y conclusiones (sección 7).

\section{Marco conceptual}

Los estudios sobre innovación han crecido dentro de la comunidad científica en los últimos años (Castellaci et al., 2005; Fagerberg, 2009). La forma en la que se crean "nuevos recursos en una situación marcada por continuos cambios en la tecnología, las preferencias y las instituciones" (Mytelka, 2000, 16), es el centro de interés de la mayoría de estos trabajos. Del mismo modo, la existencia de asimetrías en la información impulsa el cambio tecnológico (Dosi et al., 1988) y crea nuevas oportunidades de explotación de este conocimiento. Por lo tanto, considerando que la incertidumbre y el desequilibrio son "más la regla que la excepción" (Barge-Gil y Modrego-Rico, 2007, 246) se requiere de instrumentos adecuados para estudiar la complejidad de los procesos de innovación.

Por esta razón diversas disciplinas se han aproximado al estudio de este fenómeno desde diferentes puntos de vista. Por ejemplo, desde una vertiente institucional se analiza la coordinación de diferentes procesos e ideas por parte de un conjunto de organizaciones para crear nuevos productos o servicios (Galanakis, 2006). Así, se hace imprescindible contextualizar no sólo un conjunto dado de organizaciones objeto de estudio, sino su trayectoria histórica (Pinto, 2009). Esto es, se requiere de un marco conceptual tal que pueda ser adaptado a su propósito: la descripción de las funciones e interrelaciones entre diferentes organizaciones, siendo a su vez capaz de atender a su heterogeneidad.

La aparición del concepto de sistema nacional de innovación, enraizado en los primeros debates originados en el marco de la Organización para la Cooperación y el Desarrollo Económico (OCDE, en adelante) a partir de 1960 (Godin, 2007), trata de integrar muchos de los factores que habían sido previamente excluidos de los análisis económicos neoclásicos (Freeman, 1982). Desde entonces hasta ahora, sobre todo en Europa, autores como Freeman han desarrollado una influyente corriente de pensamiento en la que se enfatizan las interrelaciones entre diferentes actores e instituciones en los procesos de innovación, que permiten un enfoque sistémico de la misma. Bajo este prisma el resultado innovador de las empresas nacionales depende de un conjunto de instituciones y sus interacciones (Nelson, 1993, 4). De este modo, "el sistema nacional de innovación está constituido por elementos y relaciones que 
interactúan en la producción, difusión y uso de nuevo, y económicamente útil, conocimiento" (Lundvall, 1992, 2).

En respuesta a su inadecuación a ciertos niveles de análisis, otros autores propusieron versiones adaptadas tanto geográfica como sectorialmente. De esta forma, el enfoque de los sistemas locales de innovación propone una redefinición del espacio de estudio (hacia lo local, lógicamente) para capturar la idiosincrasia del territorio, más allá de sus límites administrativos (De la Mothe, 1998). De la misma forma, desde el punto de vista sectorial, otros autores han enfatizado las características específicas de los procesos de innovación en diferentes sectores independientemente de su localización territorial (Breschi y Malerba, 1997), definiendo el concepto de sistema sectorial de innovación y de producción como "un conjunto de productos nuevos y establecidos para usos específicos y el conjuntos de agentes que lleva a cabo interacciones tanto de mercado como de no-mercado para la creación, producción y venta de aquellos productos" (Malerba, 2002, 250). Por lo tanto, se observa que no sólo la presencia de diferentes instituciones sino también el estudio de sus interrelaciones se consideran rasgos esenciales y comunes en los conceptos anteriores. A tener en cuenta, empresas locales/globales, instituciones de educación (primaria, secundaria, profesional y superior), institutos tecnológicos, instituciones gubernamentales, cuerpos de regulación y estandarización, entre otros. En este estudio, se ha adaptado el modelo de sistema de innovación propuesto por Arnold y Kuhlmann (2001), cuya novedad con respecto a anteriores aproximaciones radica en la inclusión del sistema de demanda, imprescindible para entender la innovación en sectores de baja intensidad tecnológica, como el textil-confección (ver Gráfico 1).

Bajo este enfoque, el núcleo central de un sistema de innovación está constituido por el sistema industrial definido como un conjunto de empresas localizadas en el mismo territorio y pertenecientes a actividades económicas relacionadas, como, por ejemplo, la industria textil y sus industrias auxiliares. Dentro de este conjunto de empresas, destaca el papel que en los procesos de innovación territorial desempeñan un grupo reducido de empresas de base tecnológica que al adoptar nuevos desarrollos en otros

GRÁFICO 1. MODELO DE ANÁLISIS DEL SISTEMA SECTORIAL DE INNOVACIÓN ADAPTADO A UNA REGIÓN

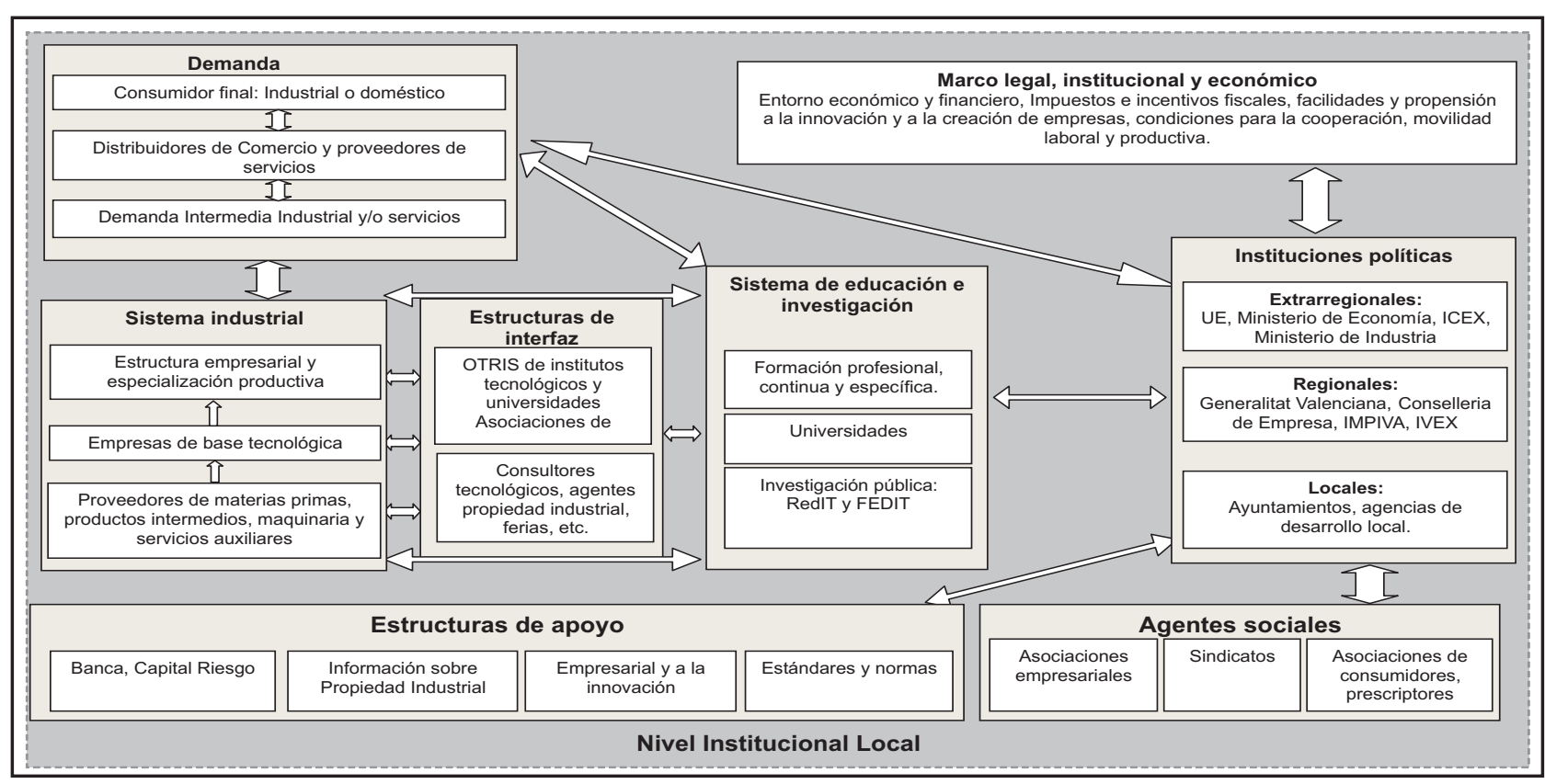

Fuente: Arnold y Kuhlmann (2001). 
campos (tecnologías de la información y la comunicación, química y nuevos materiales, maquinaria), contribuyen a la adaptación, por mimetismo, del tejido productivo (Storey y Tether, 1998).

En segundo lugar, el sistema de demanda es incluido en este marco analítico debido a su importancia en la generación de innovaciones. La mayoría de innovaciones en el sector textil-confección son prescritas por las especificaciones del mercado (Alto Consejo Consultivo, 2007, 150). Además, un análisis sobre el funcionamiento de los canales de distribución es imprescindible para comprender las dinámicas especificas del sistema de innovación, especialmente en los últimos años.

En tercer lugar, el conjunto denominado estructuras de interfaz o intermediarios está formado por todas las instituciones públicas y privadas cuyo objetivo es transferir tecnología y conocimiento al sistema industrial desde otras esferas. Dentro de esta categoria, cabe destacar las oficinas de transferencia de resultados de investigación, los institutos tecnológicos y centros de investigación, las universidades y otros agentes como las oficinas de gestión de la propiedad intelectual e industrial. La mayoría de estas infraestructuras tecnológicas y científicas desempeñan un doble papel en el sistema de innovación, puesto que no sólo proveen de activos tangibles e intangibles orientados a la innovación, sino que en la medida en la que se relacionan con los diversos agentes que integran el sistema, actúan también como instrumento clave para la articulación del mismo. Este concepto de estructura de interfaz, resulta sumamente interesante en este tipo de modelos puesto que se parte de una concepción de la innovación de carácter interactivo, en el que la cantidad, pero también la calidad de las relaciones de los agentes que lo integran, comporta efectos sobre la propia articulación del sistema de innovación (Fernández de Lucio, I. et al., 1996).

De todos los agentes que conforman el sistema de educación e investigación las universidades, en particular, están experimentando una gran transformación no sólo en lo que se refiere a la creación y transmisión de conocimiento, sino también respecto a su creciente papel en los procesos de innovación a través de las relaciones extra-académicas de sus miembros con los demás agentes (Shattock, 2005; Kitagawa, 2005). Con todo, las universidades son capaces de proveer no sólo conocimiento y tecnología, sino también personal cualificado que, a su vez, contribuye a potenciar la competitividad de las empresas locales (Bonaccorsi y Piccaluga, 1994). En este sentido, la capacidad de aprendizaje y absorción de nuevo conocimiento en las empresas dependerá de sus capacidades internas, ligadas a la cantidad de personal disponible y su cualificación científico-técnica que, en último extremo, adquiere y absorbe la tecnología. Este personal, como tal, es una fuente de acumulación y creación de conocimiento que, en última instancia, es el principal input utilizado por las empresas en su proceso innovador (Cohen y Levinthal, 1990).

Por último, la inclusión del marco legal dentro del esquema de sistema de innovación propuesto es crucial, ya que es éste el que sienta las bases y principios de actuación de los agentes y sus interrelaciones, es decir, las condiciones legales bajo las cuales se dan las dinámicas del sistema. Precisamente en los contextos de baja tecnología (en contraste con los ya ampliamente estudiados contextos de alta tecnología) el análisis de las dinámicas de generación y diseminación del conocimiento y el papel que las instituciones pueden jugar en las mismas, son clave para el entendimiento tanto de las fuentes como de los resultados de los procesos de innovación. En esta línea, algunos estudios han desarrollado una categorización de diferentes contextos cuya transformación industrial condiciona los distintos roles que, por ejemplo, la universidad (u otros organismos) juega(n) en el desarrollo local (Lester, 2005).

Si bien algunos trabajos recientes han utilizado la región (Comunitat Valenciana) como unidad de análisis para estudiar la colaboración entre industria y universidad (García Aracil y Fernández de Lucio, 2008), el presente trabajo se concentra en los procesos de innovación de un distrito industrial específico circunscrito a tres comarcas, L'Alcoià, El Comtat y La Vall d'Albaida, pertenecientes a las tradicionalmente conocidas como las Comarcas Centrales Valencianas. Para ello, se han contextualizado tanto los agentes que lo conforman como las relaciones que lo articulan. A pesar de que la ausencia de micro-datos a este nivel de análisis obstaculizaba la obtención de resultados y conclusiones, la puesta en marcha de diversos estudios empíricos llevados a cabo por los autores acerca de las fuentes de innovación y las relaciones entre agentes en esta área geográfica en los últimos años, ha mejorado el entendimiento de este campo en ciernes. 


\section{FuENTES DE INFORMACIÓN Y ENFOQUE ADOPTADO EN EL TRABAJO}

Como ya se ha mencionado en la sección anterior, este artículo analiza los procesos de innovación de la industria textil en un distrito industrial específico. A su vez, recoge los principales resultados y conclusiones de diferentes proyectos en los cuales los autores han participado entre los años 2004 y 2009. En la tabla siguiente, se muestran las principales referencias de los proyectos fuente de este artículo, incluyendo el nombre del proyecto, los principales objetivos perseguidos y el período de estudio. Todos los proyectos ya finalizados han utilizado el marco conceptual expuesto en la sección 2.

TABLA 1. PRINCIPALES PROYECTOS ACERCA DE LA INNOVACIÓN EN LA INDUSTRIA TEXTIL-CONFECCIÓN (2004-2009)

\begin{tabular}{|l|l|l|}
\hline \multicolumn{1}{|c|}{ Proyectos } & \multicolumn{1}{c|}{ Objetivos } & Período \\
\hline $\begin{array}{l}\text { Plan Estratégico Textil de las Comarcas } \\
\text { Centrales Valencianas }\end{array}$ & $\begin{array}{l}\text { Aumentar la competitividad de las empresas textiles valencianas a través de la mejora de } \\
\text { su tecnología así como de sus canales de distribución. }\end{array}$ & 2004-2005 \\
\hline $\begin{array}{l}\text { Estratex: el cambio estratégico en la PYME } \\
\text { textil }\end{array}$ & $\begin{array}{l}\text { Extender los principales resultados del proyecto anterior (Plan Estratégico Textil de las } \\
\text { Comarcas Centrales Valencianas), pero teniendo en cuenta esta vez sólo las empresas con } \\
\text { menos de } 20 \text { trabajadores. }\end{array}$ & 2006 \\
\hline $\begin{array}{l}\text { Canales de Distribución de Textiles } \\
\text { Técnicos }\end{array}$ & $\begin{array}{l}\text { Analizar y determinar los diferentes canales de distribución de los diferentes productos } \\
\text { dentro del segmento del textil técnico. }\end{array}$ & $2006-2007$ \\
\hline $\begin{array}{l}\text { La innovación en el sector textil-confección } \\
\text { de la Comunidad Valenciana }\end{array}$ & $\begin{array}{l}\text { Analizar el esfuerzo innovador realizado por las empresas que pertenecen al sector textil- } \\
\text { confección en la Comunitat Valenciana. }\end{array}$ & $2006-2007$ \\
\hline $\begin{array}{l}\text { Sistemas de innovación de textiles técnicos } \\
\text { en la Unión Europea }\end{array}$ & $\begin{array}{l}\text { Comparar cinco sistemas de innovación sectoriales diferentes relacionados con productos } \\
\text { de textil técnico en la Unión Europea. }\end{array}$ & $2007-2009$ \\
\hline
\end{tabular}

Fuente: Elaboración propia.

Con respecto a la recolección de los datos, se han utilizado diversas fuentes, entre las que cabe destacar cuestionarios y entrevistas (primarias), así como análisis de documentos y explotación de bases de datos creadas ad hoc (secundarias). En términos generales, el nivel de análisis ha cambiado ligeramente del primer al último proyecto, si bien cabe resaltar que todos ellos se han circunscrito a las empresas textiles y a sus instituciones de apoyo ubicadas en las CCV (ver figura 1). No obstante, la dimensión geográfica ha abarcado principalmente tres comarcas: L'Alcoià-El Comtat y la Vall d'Albaida; mientras que la sectorial se ha ceñido a la industria textil a la hora de delimitar el estudio. A pesar de haber tomado esta decisión, los autores de este artículo reconocen, tal como afirman Carlsson y sus colegas, la complejidad de establecer esta clase de límites en los estudios de innovación (Carlsson et al., 2002).
FIGURA 1. MAPA DE LAS COMARCAS CENTRALES VALENCIANAS

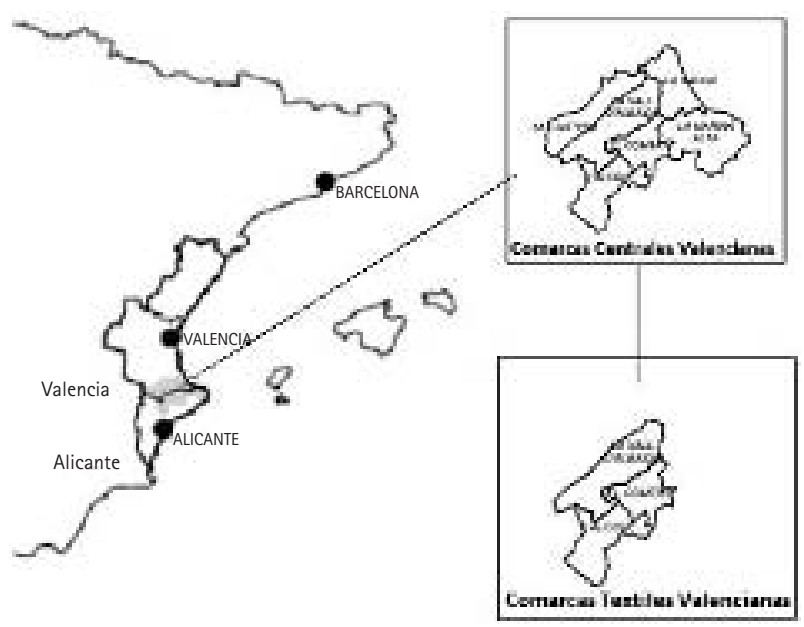

Fuente: Adaptado de OCDE (2001).

ARBOR Vol. 188753 enero-febrero [2012] 75-96 ISSN: 0210-1963 


\section{- Entrevistas}

La poca disponibilidad (y en ocasiones fiabilidad) de datos estadísticos en este tipo de estudios sobre innovación a nivel local durante las pasadas décadas (Such, 1990) ha suscitado la necesidad de trabajos de campo específicos en los cuales los investigadores interesados acuden directamente a recopilar la información pertinente. Debido al tipo de proyectos que se realizaron durante el período 20042009 (ver tabla 1), se creyó conveniente realizar entrevistas a los agentes localizados dentro del sistema territorial, con el fin no sólo de conocer de primera mano la problemática del sector, sino además informar acerca de los objetivos de cada uno de los estudios. Con el fin de concertar una cita de manera adecuada, se contactó con las personas que iban a ser entrevistadas por vía telefónica. Una vez se confirmó el día, la hora y el lugar de la entrevista se inició el trabajo de campo. En el siguiente cuadro podemos observar de manera agregada todos los agentes que participaron y que son la fuente de información de este artículo:

\begin{tabular}{|l|c|l|}
\hline \multicolumn{1}{|c|}{ Tipo de agente } & Número & \multicolumn{1}{|c|}{ Observaciones } \\
\hline Empresas & 84 & $\begin{array}{l}\text { Empresas cuya actividad económica principal es el textil- } \\
\text { confección. }\end{array}$ \\
\hline Centros de Educación e Investigación & 4 & $\begin{array}{l}\text { Universidad Politécnica de Valencia (Campus de Alcoy). } \\
\text { Escuelas de Formación Profesional (especialidad textil). } \\
\text { Escuela de Artes y Oficios de Alcoy. } \\
\text { AlTEX. }\end{array}$ \\
\hline Agentes Sociales & 2 & Sindicatos. \\
\hline Asociaciones empresariales & 3 & $\begin{array}{l}\text { ATEVAL. } \\
\text { ACETEX. } \\
\text { Textil Alcoyana. }\end{array}$ \\
\hline
\end{tabular}

Fuente: Elaboración propia.

En cuanto al tipo de entrevista que se llevó a cabo, hay que resaltar que se escogió la entrevista en profundidad (Marshall y Rossman, 1998) en donde el grupo responsable de la investigación diseñó un guión de entrevista para cada grupo de interesados. Al tratarse de grupos heterogéneos se optó por un tipo de entrevista semiestructurada que contenía algunas preguntas clave comunes para todas las personas participantes, dejando abiertas otras que irian surgiendo a lo largo de las mismas. Estos guiones contenían la lista de preguntas o temas a ser explorados durante el trabajo de campo además de incluir un formulario de consentimiento informado.

\section{- Encuestas}

Aparte de las entrevistas realizadas se distribuyó entre éstas y un grupo de 500 empresas asociadas a ATEVAL, un cuestionario estructurado que serviría para obtener datos cuantitativos de la interrelación existente entre los diferentes agentes del sistema de innovación, así como del esfuerzo innovador realizado por las empresas. En conjunto se obtuvieron 134 cuestionarios.

\section{Fuentes secundarias}

En cuanto a las fuentes secundarias, se consultaron tres tipos de literatura. En primer lugar, la que enmarca teóricamente el estudio y que se encuentra a caballo entre la literatura sobre distritos industriales y los trabajos sobre sistemas de innovación. Esta etapa sirvió tanto para plantear el presente trabajo de investigación (punto 1) como para informar al lector del marco teórico en el que se insertaba el estudio (punto 2). En segundo lugar, se revisó la literatura sobre los antecedentes históricos del área objeto de estudio, incluyendo tanto la que aglutina las principales cifras de la industria textil-confección, como la que lleva a cabo distintos análisis evolutivos de la misma (punto 4). Por último, fue necesario también incluir una revisión de la 
literatura sobre el papel de las instituciones con la finalidad de incluir en sus debates la contribución del presente trabajo: el estudio de un sistema local de innovación en una región que cuenta con la presencia de instituciones de larga trayectoria histórica basadas fundamentalmente en una industria tradicional de baja capacidad de absorción (punto 5).

\section{Caracterización de la industria textil- confección en la Comunidad Valenciana}

En 2009, la industria textil-confección valenciana representaba, en términos de producción, algo menos del 17\% del total nacional, por detrás tan sólo de Cataluña (41\%) y ligeramente por encima de Galicia (15\%). En primer lugar, a diferencia de lo que ocurre en el conjunto nacional, la especialización regional de este sector se asienta en el subsector textil [códigos de la clasificación nacional de actividades económicas (CNAE 93) 171 a 177], que supone el $67 \%$ de las empresas y el $73 \%$ del empleo sectorial, frente al $44 \%$ y $47 \%$ en el conjunto nacional, respectivamente. Esta especialización productiva, como ya se ha indicado, está vinculada a la enorme tradición en la producción de artículos de textil-hogar en las comarcas de la Vall d'Albaida, L'Alcoià y El Comtat, en las que se reproduce, hasta cierto punto y salvando las distancias, el modelo de distrito industrial italiano, donde algunas empresas se interconectan a lo largo de la cadena de valor y cada una de ellas se especializa en una parte del proceso de producción.

TABLA 2. PRINCIPALES VARIABLES ECONÓMICAS DE LA INDUSTRIA TEXTIL-CONFECCIÓN EN ESPAÑA Y EN LA COMUNITAT VALENCIANA (2008)

\begin{tabular}{|c|c|c|c|c|c|c|}
\hline & $\begin{array}{c}\text { Total } \\
\text { industria }\end{array}$ & $\begin{array}{l}\text { Fibras, hilos } \\
\text { y tejidos }\end{array}$ & $\begin{array}{l}\text { Acabado } \\
\text { de textiles }\end{array}$ & $\begin{array}{c}\text { Otras } \\
\text { industrias } \\
\text { textiles }\end{array}$ & $\begin{array}{l}\text { Géneros } \\
\text { de punto }\end{array}$ & Confección \\
\hline \multicolumn{7}{|l|}{ COMUNITAT VALENCIANA } \\
\hline $\begin{array}{l}\text { Número de empresas según la actividad } \\
\text { de la empresa }\end{array}$ & 20.869 & 349 & 154 & 747 & 116 & 672 \\
\hline Personas ocupadas & 321.257 & 5.018 & 2.979 & 8.772 & 1.658 & 6.313 \\
\hline Ingresos de explotación (miles euros) & 64.489 .987 & 655.535 & 285.239 & 997.110 & 125.903 & 458.258 \\
\hline Inversión en activos materiales (miles euros) & 2.578 .626 & -4.530 & 14.169 & 28.768 & 1.853 & 13.513 \\
\hline \multicolumn{7}{|l|}{ ESPAÑA } \\
\hline $\begin{array}{l}\text { Número de empresas según la actividad } \\
\text { de la empresa }\end{array}$ & 151.320 & 940 & 704 & 2.888 & 982 & 6.983 \\
\hline Personas ocupadas & 2.580 .378 & 17.797 & 9.997 & 31.142 & 11.950 & 79.757 \\
\hline Ingresos de explotación (miles euros) & 636.397 .600 & 2.479 .165 & 956.699 & 3.531 .744 & 1.236 .633 & 7.661 .888 \\
\hline Inversión en activos materiales (miles euros) & 28.120 .833 & 23.451 & 27.139 & 150.244 & 19.273 & 174.107 \\
\hline \multicolumn{7}{|l|}{$\%$ COMUNITAT VALENCIANA/ESPAÑA } \\
\hline $\begin{array}{l}\text { Número de empresas según la actividad } \\
\text { de la empresa }\end{array}$ & 13,79 & 37,13 & 21,88 & 25,87 & 11,81 & 9,62 \\
\hline Personas ocupadas & 12,45 & 28,20 & 29,80 & 28,17 & 13,87 & 7,92 \\
\hline Ingresos de explotación & 10,13 & 26,44 & 29,81 & 28,23 & 10,18 & 5,98 \\
\hline Inversión en activos materiales & 9,17 & $-19,32$ & 52,21 & 19,15 & 9,61 & 7,76 \\
\hline
\end{tabular}

Fuente: Encuesta Industrial de Empresas. IVE (2010). 
TABLA 3. ESPECIALIZACIÓN PRODUCTIVA DE LOS PRINCIPALES MUNICIPIOS TEXTILES VALENCIANOS (2008)

\begin{tabular}{|c|c|c|c|c|}
\hline MUNICIPIOS & Empleo & Textil-confección & CE nacional & CE regional \\
\hline España & 20.257 .600 & 82.264 & 1 & - \\
\hline Comunitat Valenciana & 2.226 .200 & 15.007 & 1,7 & 1,0 \\
\hline Agullent & 1.195 & 850 & 175,2 & 105,5 \\
\hline Aielo de Malferit & 562 & 136 & 59,6 & 35,9 \\
\hline Albaida & 1.117 & 589 & 129,8 & 78,2 \\
\hline Alcoi & 6.185 & 972 & 38,7 & 23,3 \\
\hline Atzeneta d'Albaida & 180 & 50 & 68,4 & 41,2 \\
\hline Banyeres de Mariola & 1.353 & 782 & 142,3 & 85,7 \\
\hline Bocairent & 565 & 199 & 86,7 & 52,2 \\
\hline Canals & 987 & 110 & 27,4 & 16,5 \\
\hline Cocentaina & 2.117 & 697 & 81,1 & 48,8 \\
\hline Enguera & 364 & 84 & 56,8 & 34,2 \\
\hline Montaverner & 167 & 59 & 87,0 & 52,4 \\
\hline Muro d'Alcoi & 1.404 & 677 & 118,7 & 71,5 \\
\hline Ontinyent & 5.843 & 1.608 & 67,8 & 40,8 \\
\hline
\end{tabular}

Fuente: Elaboración propia desde datos de SABI (2010).

Como puede observarse en la tabla 3 , la especialización sectorial en los municipios de las CCV resulta sustancialmente más elevada que en el conjunto de España y la Comunitat Valenciana. En este caso, la mayoría de municipios localizados en las CCV presentan índices de especialización significativamente superiores, no sólo comparándolos con los datos a nivel nacional ( 3 . $^{a}$ columna) sino también en términos regionales (4. ${ }^{\text {a }}$ columna), destacando Banyeres de Mariola, Agullent y Albaida.

De hecho, el nacimiento de la actividad industrial textil en la Comunitat Valenciana puede ubicarse en el área de Alcoi y sus alrededores (Vallés, 1986). Algunos accidentes geográficos, como una serie de montañas escarpadas, dificultaron el desarrollo de la agricultura, que unida al uso y dominio de la fuerza hidráulica condujeron a una temprana aparición (siglo $\mathrm{X}$ y $\mathrm{XI}$ ) de actividades de origen artesanal de carácter textil (Gabinete Sigma, 1974). Ya a comienzos del siglo XIX, la introducción de maquinaria condujo al despegue de la industria del textil y del papel.
Así, surge un modelo de ciudad capitalista originado por el desplazamiento del centro de producción desde las casas particulares a la fábrica, y la cada vez más marcada estructura de clases, con una burguesía industrial pujante y fuertemente articulada, y una clase obrera abundante, viviendo en condiciones precarias.

A lo largo de los siglos XIX y XX, un gran número de recesiones cíclicas afectaron a la evolución y especialización productiva local, si bien fue el incremento de la construcción de hogares y el éxodo desde las zonas rurales a las urbanas, observado desde 1960 hasta 1975, en España, lo que ayudó a consolidar la industria textil, generando un abandono progresivo de actividades más tradicionales como la pañería y la confección. Durante el proceso de reconversión, el cambio tecnológico fue posible gracias a la importación de maquinaria desde países como Alemania, Italia y Suiza, lo que supuso la desaparición de la industria local de construcción de maquinaria textil. Este proceso junto a la abundancia de 
mano de obra barata permitió aumentar la competitividad no sólo en el mercado nacional, sino también en el internacional. Desde este periodo las empresas fueron especializándose en el nicho de mercado del textil para el hogar cuyos principales productos eran mantas, toallas, trapos y cortinas, entre otros.

\section{Caracterización del sistema de innovación TEXTIL EN LAS CCV}

\subsection{El territorio}

Las CCV han sido tradicionalmente definidas como un distrito industrial especializado en sectores maduros, principalmente en la industria textil. En uno de los pocos trabajos enfocados al estudio de estas comarcas, la Organización para la Cooperación y el Desarrollo Económico la calificó como región intermedia, caracterizada por una distribución geográfica uniforme de numerosas ciudades y pueblos a lo largo del territorio, cuyas dinámicas de desarrollo económico y social están sujetas a las fluctuaciones derivadas de la desaceleración en su sector manufacturero principal (OCDE, 2001).

Una característica diferencial de los territorios de las CCV radica en el elevado peso de la industria en La Vall d'Albaida, L'Alcoià y El Comtat, respecto al total regional, y el reducido peso del sector servicios. Esta estructura económica se ha convertido en los últimos años en un problema añadido para el desarrollo local, dado que, por una parte, la creciente deslocalización de procesos de fabricación hacia países de bajo coste de mano de obra ha desarticulado la configuración tradicional del sistema productivo local, y, por otra, porque la escasa dotación de servicios dificulta la atracción de trabajadores con elevados niveles de cualificación, necesarios en un momento de cambio estructural.

Además, ante la pérdida paulatina de competitividad en el sector textil, algunas de las empresas más significativas del sector llevaron a cabo un proceso de diversificación hacia la construcción, al albur de la burbuja inmobiliaria, reduciéndose drásticamente la inversión en activos materiales (un 41\% inferior en 2008 que en 2003, en la Comunidad Valenciana, frente a un 36\% en España). Esta caída en la renovación tecnológica se explica no sólo por las malas perspectivas sectoriales (tabla 4), sino también porque el comportamiento del sistema financiero local favorecía actuaciones de mera especulación inmobiliaria, que garantizaban un tiempo de recuperación muy corto, y la propia expansión del negocio bancario mediante la extensión del crédito inmobiliario. Unido a los factores presentados anteriormente hay que destacar la percepción negativa generalizada del sector en su conjunto (ver tabla 4), síntoma, sin duda, de un inminente ajuste estructural en el mismo.

TABLA 4. PERCEPCIÓN DEL FUTURO SECTORIAL

\begin{tabular}{|l|c|c|c|c|c|}
\hline \multicolumn{1}{|c|}{ Actividad } & Muy pesimista & Pesimista & Sin cambios & Optimista & Muy optimista \\
\hline Sectores Auxiliares & $50,00 \%$ & $16,67 \%$ & $16,67 \%$ & $16,67 \%$ & $0,00 \%$ \\
\hline Hilatura & $57,14 \%$ & $21,43 \%$ & $7,14 \%$ & $14,29 \%$ & $0,00 \%$ \\
\hline Tejeduría & $18,75 \%$ & $56,25 \%$ & $25,00 \%$ & $0,00 \%$ & $0,00 \%$ \\
\hline Acabados & $12,50 \%$ & $75,00 \%$ & $0,00 \%$ & $0,00 \%$ & $12,50 \%$ \\
\hline Art. Textiles & $13,16 \%$ & $36,84 \%$ & $39,47 \%$ & $10,53 \%$ & $0,00 \%$ \\
\hline Otros Textiles & $3,13 \%$ & $25,00 \%$ & $40,63 \%$ & $15,63 \%$ & $\mathbf{1 5 , 6 3} \%$ \\
\hline Género Punto & $33,33 \%$ & $25,00 \%$ & $33,33 \%$ & $8,33 \%$ & $0,00 \%$ \\
\hline Confección & $50,00 \%$ & $12,50 \%$ & $25,00 \%$ & $12,50 \%$ & $0,00 \%$ \\
\hline Total general & $\mathbf{2 1 , 6 4} \%$ & $\mathbf{3 3 , 5 8} \%$ & $\mathbf{2 9 , 8 5 \%}$ & $\mathbf{1 0 , 4 5 \%}$ & $\mathbf{4 , 4 8} \%$ \\
\hline
\end{tabular}

Fuente: Elaboración propia mediante encuesta a 134 empresas, realizada en 2008. 


\subsection{Sistema industrial}

La clasificación de la industria textil se puede realizar (gráfico 2) en base a: 1) las fibras usadas (inputs), 2) las diferentes fases del proceso productivo y 3) los usos de los productos finales (outputs).

Las fibras son la principal materia prima de la industria textil, si bien, debido al exceso de demanda, las fibras naturales han sido progresivamente sustituidas por las sintéticas. Estas últimas están vinculadas a la industria quimica y su desarrollo ha permitido añadir nuevas funcionalidades a los productos textiles tradicionales, siendo sus principales proveedores empresas multinacionales con sede principal en Estados Unidos, Europa (por ejemplo, DuPont) y Japón.

El sector químico también desempeña un papel determinante como proveedor de otras materias primas, como son los tintes o los blanqueantes y aprestos. De nuevo, se observa un control en la provisión de estos productos por parte de corporaciones multinacionales, como BASF o Bayer. Finalmente, otro sector clave en la industria textil, el sector de la maquinaria, engloba un conjunto de proveedores muy heterogéneo de acuerdo a diferentes niveles de complejidad en los procesos. Tradicionalmente ha habido en España un conjunto de empresas importantes de maquinaria textil, localizadas en el área de Cataluña (Alto Consejo Consultivo, 2007), si bien, durante los últimos años se ha producido un progresivo abandono de este sector, incapaz de competir con las importaciones de empresas alemanas e italianas, y más recientemente con las de origen chino. No obstante, en el caso de las CCV la paulatina desaparición de la industria de maquinaria local, llevó a la revalorización de las actividades de muchos talleres locales de reparación y servicios de mantenimiento de maquinaria, que en la actualidad realizan procesos de ajuste puntual en las mismas. Este tipo de empresas, junto al conocimiento que tienen muchos encargados de maquinaria de las propias empresas, conocidos en el distrito como los manyàs, constituyen uno de principales mecanismos de ajuste tecnológico utilizados en las empresas (Alto Consejo Consultivo, 2007, 40). De hecho, como señalan Santamaría et al. (2009: 514), "el diseño, el uso y ajuste de maquinaria avanzada y la formación son factores decisivos para los resultados de innovación" en sectores de baja tecnología.

A pesar de la ausencia en las CCV de sectores como la producción de fibras, la industria química textil y la maquinaria, la elevada concentración de actividades espe-

GRÁFICO 2. INPUTS, PROCESO DE FABRICACIÓN Y OUTPUTS EN EL SECTOR TEXTIL-CONFECCIÓN

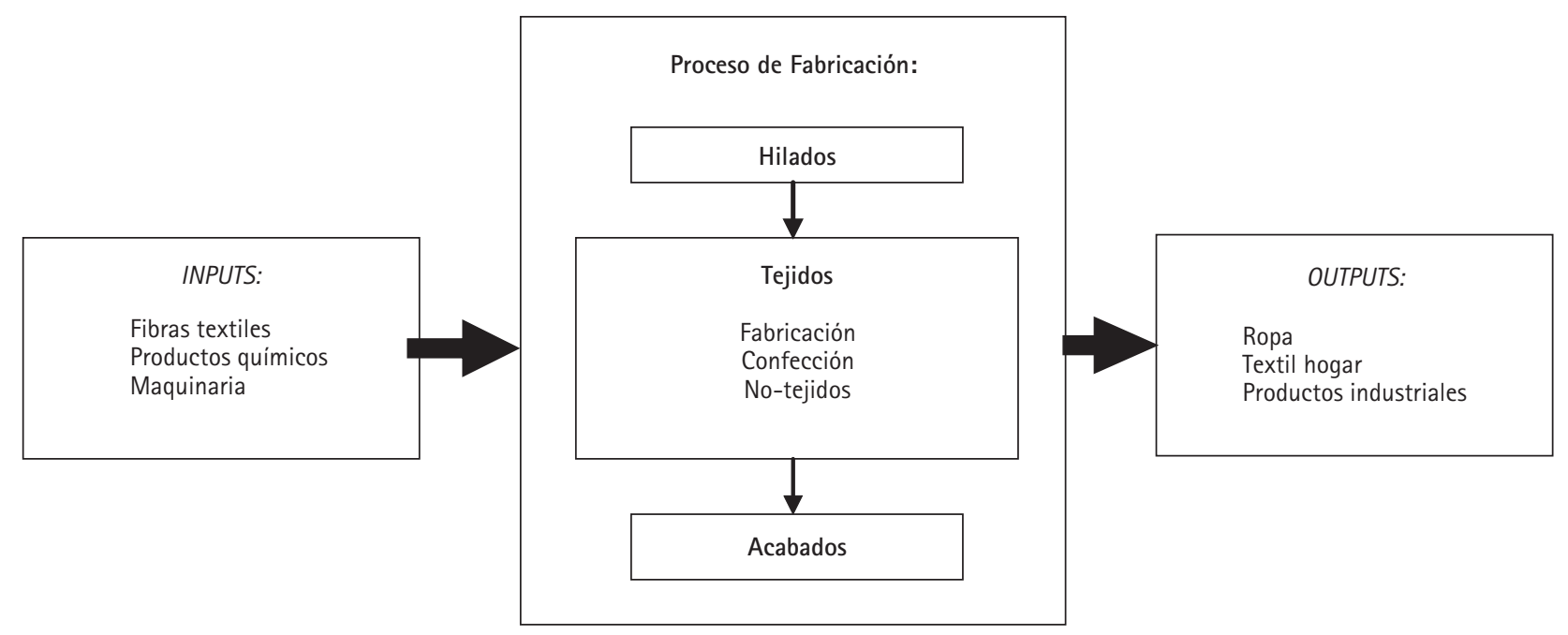

Fuente: Golf-Laville et al. (2005: 21). 
cializadas a lo largo de toda la cadena de valor, desde los textiles de cabecera (hilatura y acabados), hasta las fases finales (tejeduría, confección textil, otros productos textiles, punto y confección), configura territorialmente este sector como un distrito industrial o un cluster, según la literatura (Ybarra, 1991; Capó-Vicedo et al., 2008). La ventaja de este tipo de organizaciones espaciales radica en la puesta en común de los conocimientos y capacidades técnico-productivas presentes en el territorio, reduciendo la rigidez productiva existente, por ejemplo, en las grandes empresas altamente integradas. Esta configuración espacial y productiva en torno a pequeñas y medianas empresas, dota de flexibilidad y capacidad de respuesta rápida a los cambios en el mercado, confiriendo, además, ventajas derivadas de la concentración de actividades y servicios especializados, tanto públicos, como privados. De este modo, el sector productivo se beneficia de la externalización de ciertas fases de la cadena de valor, a la vez que la demanda conjunta favorece la reducción de costes propiciados por la existencia de economías de escala y de alcance en ciertas fases productivas, sin olvidar los efectos de desbordamiento de conocimiento (spillover) que impulsan la renovación de productos y procesos en el conjunto de empresas (Paci y Usai, 1999).

De este modo, a pesar de que a nivel local tradicionalmente existe un número muy elevado de empresas compitiendo en los mismos segmentos de mercado de producto final 0 procesos productivos, la formación de acuerdos tácitos y explícitos, generalmente basados en relaciones de confianza personal entre productores y demandantes, impulsa la competitividad a través del aprovechamiento de la capacidad productiva conjunta del territorio. Así, por ejemplo, un principio claro que tácitamente ha contribuido al mantenimiento de estas relaciones en el distrito de textil de las CCV se derivaba del mantenimiento de la confidencialidad por parte de las empresas ubicadas en los segmentos de cabecera, principalmente la de acabados, y de la autocontención de la integración vertical hacia adelante, para no entrar en competencia con los clientes finales.

Este tipo de acuerdos posibilitó una fuerte extensión tanto de la industria de acabados y tintes, cuyo grado de conexión con el sector químico resulta muy elevado $y$, que, además, debido a la gran acumulación de pedidos por parte de las fases de hilatura y tejeduría, posee un conocimiento crucial de las innovaciones de productos y procesos rea- lizados en el conjunto de la industria. En el caso de las CCV, esta industria más intensiva en conocimiento, y la hilatura cuyo grado de mecanización resulta más elevado que la media, haciendo compleja la entrada debido al esfuerzo inversor, presentan históricamente los tamaños medios empresariales más elevados (20 trabajadores en acabados y tintes, frente a 11 en otras industrias textiles, según datos de 2008). De este modo, la acumulación de pedidos permitía la obtención de economías de escala, de las que se beneficiaba el conjunto del sector.

No obstante, la irrupción de la crisis textil, desde 2003, ha llevado a una nueva configuración sectorial derivada del creciente grado de integración de alguna de las industrias de acabado más relevantes del distrito (Colortex y Moltó Reig, por ejemplo) que ante la caída en el volumen de pedidos debido no sólo a la paulatina pérdida de mercado de las empresas ubicadas en el segmento de textil-hogar, sino también a la tendencia creciente a importar productos acabados y semiacabados (tanto tejeduría, como hilatura), han comenzado a integrar un número creciente de procesos tanto de hilatura, como de tejeduría, rompiendo las relaciones de confianza que tradicionalmente han articulado el distrito (Golf-Laville et al, 2008, 167). Así, esta caída en la demanda de algunos procesos realizados en el interior del distrito ha introducido formas destructivas de competencia en precios entre algunos subcontratistas locales, que únicamente se ha visto compensada para los grupos con mejor posicionamiento en el mercado, por el aumento de la importancia de las relaciones basadas en la confianza personal entre las industrias contratistas y subcontratistas, al igual que ocurrió en el distrito industrial de Prato (Italia) durante la crisis de los ochenta (Dei Ottati, 2005). Del mismo modo, al igual que en las industrias de cabecera ha habido una tendencia creciente hacia la integración vertical, en las de tejeduría y textil-hogar se ha producido tanto una diversificación hacia el segmento de los textiles técnicos (automoción, construcción, uso público, etc.), como una mayor integración de productos de textil-hogar en catálogo, llegando incluso a la fabricación de pañería para el sector de la confección. De hecho, como se aprecia en la tabla 5, las estrategias con mayor orientación al mercado (incorporación de mayor diseño, creación de marca propia y de tiendas propias) y la diversificación hacia el segmento de los textiles técnicos constituian las que alcanzaban mayor relevancia entre el grupo de 134 empresas encuestadas.

ARBOR Vol. 188753 enero-febrero [2012] 75-96 ISSN: 0210-1963 
TABLA 5. VALORACIÓN DE ESTRATEGIAS EMPRESARIALES (\%)

\begin{tabular}{|c|c|c|c|c|c|}
\hline & Nada importante & Poco importante & Importante & Bastante importante & Muy importante \\
\hline Diversificación textil técnico & 42,54 & 6,72 & 14,18 & 8,96 & 27,61 \\
\hline Incorporación de prod. importado & 44,78 & 14,18 & 21,64 & 10,45 & 8,96 \\
\hline Deslocalización/multilocalización & 99,25 & 0,00 & 0,75 & 0,00 & 0,00 \\
\hline Sustitución producto importado & 44,03 & 21,64 & 19,40 & 7,46 & 7,46 \\
\hline Creación Marca & 31,34 & 5,97 & 15,67 & 16,42 & 30,60 \\
\hline Franquicias producto & 73,13 & 14,18 & 7,46 & 4,48 & 0,75 \\
\hline Tiendas propias & 61,19 & 11,94 & 7,46 & 6,72 & 12,69 \\
\hline Mayor diseño & 38,81 & 1,49 & 13,43 & 11,94 & 34,33 \\
\hline Fusión empresas & 52,99 & 13,43 & 15,67 & 9,70 & 8,21 \\
\hline Adquisición empresas & 69,40 & 8,21 & 14,18 & 2,99 & 5,22 \\
\hline Diversificación no relacionada & 55,97 & 13,43 & 21,64 & 2,99 & 5,97 \\
\hline Abandono actividad & 81,34 & 11,19 & 3,73 & 2,24 & 1,49 \\
\hline
\end{tabular}

Fuente: Elaboración propia mediante encuesta a 134 empresas, realizada en 2008.

Por lo que a las variables relativas al esfuerzo innovador desarrollado por el sector se refiere, cabe indicar que tanto a nivel sectorial como a nivel local existe una carencia de datos oficiales. Sin embargo, partiendo de los trabajos de campo realizados en los últimos años, en la tabla 6 se han representado algunas de las variables relevantes tanto del esfuerzo innovador realizado por las empresas, como de sus recursos y capacidades.

TABLA 6. VARIABLES DEL ESFUERZO INNOVADOR POR TAMAÑO DE EMPRESA

\begin{tabular}{|l|c|c|c|c|c|}
\hline $\begin{array}{c}\text { Tamaño empresa } \\
\text { (Número de } \\
\text { empleados) }\end{array}$ & $\begin{array}{c}\text { I+D continua } \\
\text { (tres años) }\end{array}$ & $\begin{array}{c}\text { \% Titulados } \\
\text { superiores } \\
\text { (tres años) }\end{array}$ & $\begin{array}{c}\text { \% Media de los } \\
\text { gastos en I+D/Ventas } \\
\text { (tres años) }\end{array}$ & $\begin{array}{c}\text { Cooperación para } \\
\text { I+D+I } \\
\text { (tres años) }\end{array}$ & $\begin{array}{c}\text { Frecuencia } \\
\text { protección industrial } \\
\text { (media)* }\end{array}$ \\
\hline $1-9$ & $20,00 \%$ & 26,89 & 5,77 & $36,00 \%$ & 1,04 \\
\hline $10-19$ & $26,92 \%$ & 22,10 & 2,98 & $30,77 \%$ & 1,19 \\
\hline $20-49$ & $24,39 \%$ & 11,85 & 3,03 & $36,59 \%$ & 1,39 \\
\hline $50-99$ & $46,15 \%$ & 16,60 & 5,35 & $61,54 \%$ & 1,08 \\
\hline $100-199$ & $50,00 \%$ & 11,19 & 2,19 & $66,67 \%$ & 1,83 \\
\hline$>200$ & $50,00 \%$ & 7,96 & 0,75 & $25,00 \%$ & 1,25 \\
\hline Total & $\mathbf{3 1 , 3 4 \%}$ & $\mathbf{1 2 , 9 6}$ & $\mathbf{3 , 8 4}$ & $\mathbf{4 2 , 5 4 \%}$ & 1,26 \\
\hline
\end{tabular}

* Nota: Valores medios entre 1 (nunca) y 4 (siempre).

Fuente: Elaboración propia mediante encuesta a 134 empresas, realizada en 2008. 
La primera conclusión derivada de los pocos datos oficiales existentes y de esta base de datos elaborada ad hoc es que los empresarios textiles afirman que su inversión real en actividades de I+D supera las cifras ofrecidas por la Encuesta sobre innovación oficial. Sin embargo, esta conclusión debe de ser entendida teniendo en cuenta que probablemente sólo aquellas empresas que hayan llevado a cabo este tipo de actividades consideraron útil esta clase de estudios $y$, por lo tanto, decidieron participar en el mismo. De hecho, destacan también los elevados porcentajes de empresas que realizaban I+D continua, cooperación con otras empresas para la I+D+I, así como los elevados porcentajes de titulados superiores.

A la luz de estos datos cabe destacar que la inversión en I+D de las empresas está claramente correlacionada con el tamaño, a pesar de que el conjunto de empresas con más de 200 trabajadores registró niveles inferiores de inversión dedicados a este fin. También los bajos valores medios registrados respecto a la frecuencia con la que las empresas realizan protección industrial (1. Nunca; 2 . Rara vez), subraya lo indicado anteriormente respecto a los aspectos formales, más que funcionales, que caracterizan la innovación de este sector.

\subsection{Sistema de demanda}

Las condiciones de la demanda influyen enormemente en la generación de innovaciones en el sector textil-confec- ción. Más allá de la I+D formal y las inversiones en tecnología per se, la capacidad para vender los productos de este sector, unida a la capacidad de gestionar el diseño del producto transfiriendo todos los requerimientos necesarios a lo largo de la cadena de valor son cruciales para los resultados de innovación. Estos resultados están basados en adaptaciones tanto organizacionales como funcionales a los cambios en el entorno. A modo de ejemplo, es representativo destacar que algunas de las innovaciones más relevantes de los últimos años en los sectores de la moda y la decoración están relacionadas con innovaciones a lo largo del posicionamiento de la cadena de valor sectorial. De este modo, la introducción de conceptos como la distribución de ciclo corto, adoptada por las grandes cadenas especializadas (Inditex, H\&M, C\&A, Mango, Cortefiel, etc.) y el control creciente de los mejores puntos de venta ha supuesto un cambio radical en la capacidad para introducir innovaciones en el mercado por parte de las industrias textiles.

Como se aprecia en el gráfico 3, desde los años ochenta se ha producido una progresiva disminución de las ventas en las cadenas minoristas multimarca, que constituian la principal base de la demanda de los productos textiles tradicionales, mientras el formato de la gran distribución organizada y el de hipermercados han alcanzado crecimientos espectaculares. Huelga decir que este tipo de distribución presenta ventajas en costes derivadas del

GRÁFICO 3. EVOLUCIÓN DE LA DISTRIBUCIÓN TEXTIL-CONFECCIÓN EN ESPAÑA

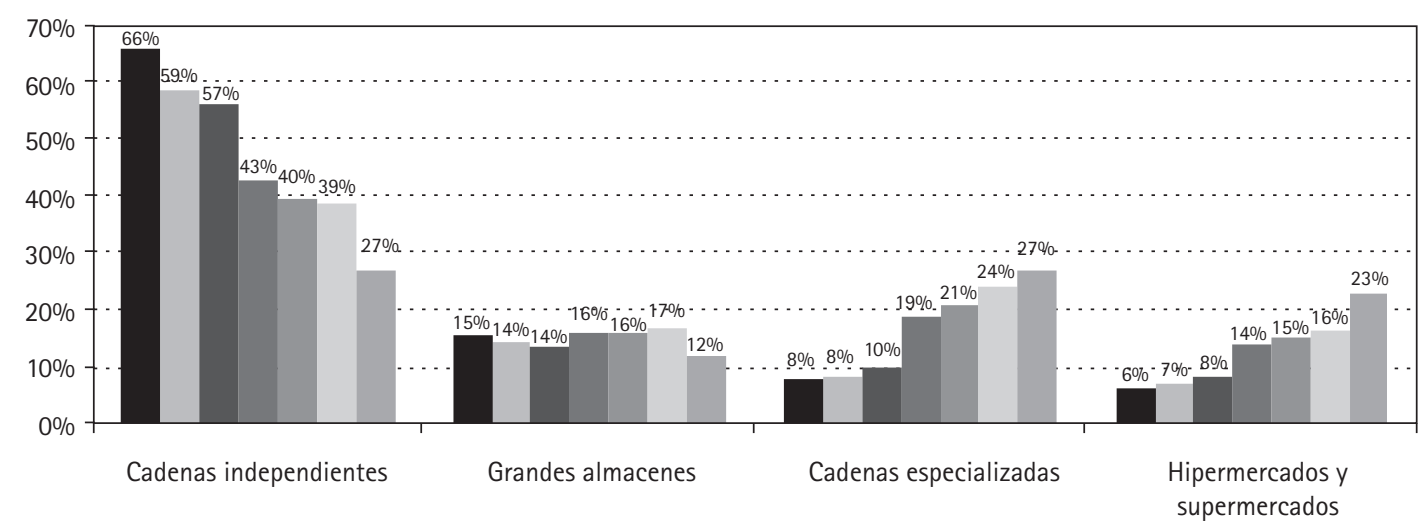

ロ1985 $\square 1994 \quad \square 1995 \quad \square 1999 \quad \square 2001 \quad \square 2003 \quad \square 2008$

Fuente: Acotex, 2009 
mayor volumen de pedidos y de la gestión más eficiente de la logística y los stocks, apoyada en la introducción de potentes sistemas informáticos que conectan el punto de venta con los almacenes o fábricas centrales. La creciente concentración del poder de mercado de estas empresas ha encontrado en la liberalización mundial de los intercambios de textil-confección, el complemento perfecto para explotar economías de escala mediante la subcontratación en países de bajo coste de procesamiento. Su gestión actual de la cadena de valor se focaliza en centralizar las fases de diseño de producto, marketing y distribución, estableciendo control exhaustivo de costes, calidades y plazos de entrega (Alto Consejo Consultivo, 2007, 81-84).

De este modo, la creciente complejidad de entrada en el mercado para la gran mayoría de las pymes textiles, debido al creciente control de la distribución organizada, junto con la especialización histórica en la innovación de procesos, más que de productos, ha generado una tendencia a diversificar hacia el subsector de textiles técnicos, cuya definición se efectúa por cubrir funciones distintas de la de vestir y decorar, o por los mercados hacia los cuales se orien$\tan$ (Golf-Laville et al., 2008, 18). Actualmente, el proceso de diversificación hacia los textiles técnicos es una de las principales estrategias adoptadas por las empresas textiles tradicionales. Sin embargo, la falta de conexiones con los sectores de aplicación de esa demanda, como la construcción civil, el sector de la automoción, los textiles médicos, ha dificultado la introducción de nuevos productos en el mercado por parte de muchas empresas del sector. Especialmente, los textiles de hogar técnicos se han encontrado con diferentes obstáculos a la hora de introducirse en los canales de distribución convencionales, controlados de forma creciente por grandes cadenas de distribución especializadas (Zara Home, Ikea, Leroy Merlin, etc.). Del mismo modo, la introducción en sectores con mayor componente tecnológico está resultando muy lenta debido tanto a la ausencia de sectores tractores de la demanda en el entorno inmediato de las empresas, como a la escasa dotación de recursos, especialmente humanos, que pudieran aplicar los conocimientos sobre fabricación textil a los sectores de aplicación (aeronáutica, obra civil, filtración, medicina, etc.) (Golf Laville, 2008, 166).

\subsection{Sistema de educación e investigación}

Conviene tener presente que uno de los principales problemas que presenta la dinámica innovadora en este tipo de sectores tradicionales está relacionado con la propia restricción de recursos (humanos y financieros principalmente) de la que parten las pymes que integran esta industria, pero también con la propia dependencia de las innovaciones llevadas a cabo en los sectores proveedores de materias primas y maquinaria, como ya se indicó en el apartado 1. Son además este tipo de sectores (los Ilamados de baja intensidad tecnológica, por tener un gasto de $\mathrm{I}+\mathrm{D}$ reducido con respecto a su cifra de ventas) los que acaban absorbiendo los avances en la industria de la alta tecnología (Roberson y Smith, 2009).

\section{a) Instituciones de educación superior}

Tradicionalmente, las CCV han disfrutado de una buena infraestructura educacional. Ello se deriva de que en la mayoría de localidades se cuente con escuelas de formación profesional con módulos específicos relacionados con la industria textil. De entre ellos destaca la Escuela de Artes y Oficios de Alcoy, por ser la más antigua escuela de formación profesional en la región. Actualmente este centro ofrece dos cursos sobre diseño de indumentaria e ilustración textil. Sin embargo, en la mayoría de centros de formación profesional de las CCV las titulaciones textiles más relacionadas con procesos (hilatura, ennoblecimiento, géneros de punto) han dejado de impartirse ante la falta de alumnado, desde el año 2005.

En lo que a educación superior se refiere, la Escuela Politécnica Superior de Alcoy (EPSA) ha sido y es el principal actor de la zona. Sin embargo, los bajos niveles de matriculación en los últimos años de la Ingeniería Técnica Industrial, en la especialidad de Textil, han conducido a la supresión de la titulación para el curso 2010-2011, entrando por tanto esta carrera en un proceso formal de extinción. Para hacer frente a esta situación, las asignaturas han sido repartidas entre otros grados de ingeniería industrial (Química, Mecánica y Diseño Industrial, por ejemplo) las cuales ofertarán itinerarios de especialización en Textil.

Los antecedentes de esta Escuela se remontan a la segunda mitad del siglo XIX, fecha en la que los industriales alcoyanos ante la resistencia a la introducción de maquinaria por parte de los obreros textiles (revueltas antimaquinistas, desde 1849), deciden crearla, para a través de la formación romper la resistencia a la modernización sectorial. Tras un sinfín de vaivenes y de cambios de legislación, nombre y estructura ${ }^{1}$ en 1972 se adscribe a la Universidad Politécnica de Valencia experimentando los cambios más relevantes de su historia 
tanto en términos cualitativos como cuantitativos. A modo de ejemplo, cabe destacar que si bien en sus primeros años ofertaba cinco Ingenierías Técnicas Industriales (Química, Mecánica, Electricidad, Electrónica y Textil) durante los años noventa del pasado siglo incorporó hasta seis titulaciones más (incluyendo dos superiores y un Tercer Ciclo en Textil) pasando a duplicar el número de alumnos matriculados, así como el número de profesores y personal de administración que en sus inicios era exiguo. Es además a partir de la década de los noventa del pasado siglo cuando se presencia un cambio sustancial en el papel local de este centro.

En primer lugar, se produce un aumento en el número de alumnos, que lleva consigo un aumento tanto del profesorado, como del personal de administración. El nuevo profesorado, apoyado por los cambios legislativos en torno a la educación superior, incorpora a su tradicional función docente, la labor de investigación y la de servicio al entorno productivo. En segundo lugar, y como consecuencia del primer cambio, se hace más visible la presencia de la EPSA en el territorio, que hasta entonces estaba "cerrada a la sociedad y al servicio de unas pocas familias" (cita textual extraída de las entrevistas). De hecho, uno de los mecanismos de interrelación con otros agentes locales se concretó con la creación del Área de Relaciones con el Entorno. En la misma, diversos servicios se han ido ofertando para estrechar más la vinculación del personal universitario, así como del alumnado, con las distintas organizaciones de su entorno: principalmente empresas, pero también asociaciones, ayuntamientos, institutos de investigación y fundaciones, siguiendo el modelo desarrollado en la Universidad Politécnica de Valencia. Uno de los servicios de esta reciente área de la EPSA que mejor aceptación ha tenido por parte del tejido empresarial es el servicio de prácticas en empresa y empleo, que consiste en hacer de intermediario entre la Universidad y sus alumnos y las empresas recogiendo una serie de proyectos tanto desde el punto de vista de la oferta como de la demanda. Todos los estudiantes de la EPSA tienen como requisito para finalizar sus estudios la realización de un Proyecto Final de Carrera. La mayoría de ellos intenta poner en aplicación su conocimiento teórico, aprendido durante sus estudios, en un proyecto real dentro de una empresa. Desde 2005 esta clase de interrelación entre estudiantes e industria es además económicamente recompensada (con un mínimo estipulado en $300 €$ al mes). Casi el 20\% del total de los proyectos en 2007 fueron desarrollados en empresas textiles.

En general, los empresarios textiles perciben positivamente la formación que los alumnos reciben en la EPSA, pero tienen algunas dificultades para encontrar mano de obra cualificada en este sector. Esto se debe a que pocos estudiantes escogen estos estudios (Ingeniería Técnica Industrial, especialidad en Textil) por su percepción negativa con respecto a las perspectivas futuras del sector que ya hemos apuntado anteriormente. Por ello, el hecho de desarrollar un proyecto (real) dentro de una empresa constituye una herramienta adecuada para compartir la responsabilidad de la formación del alumno entre la universidad y la industria en un momento en el que el contexto atraviesa una situación delicada en materia de perspectivas de negocio y de cambios estructurales. No obstante, como se observa en la tabla 7, existe un elevado grado de desconocimiento entre el empresariado textil de las CCV sobre los servicios que ofrece este Campus.

TABLA 7. CONOCIMIENTO DE LOS SERVICIOS DE LA EPSA Y SU UTILIZACIÓN POR PARTE DE LAS EMPRESAS

\begin{tabular}{|l|c|c|c|}
\hline \multicolumn{1}{|c|}{ Tamaño empresarial } & Sí conoce serv. universidad & Sí utiliza serv. universidades & Papel de la universidad* $^{* 1}$ \\
\hline $1-9$ & $40,00 \%$ & $8,00 \%$ & 2,76 \\
\hline $10-19$ & $34,62 \%$ & $15,38 \%$ & 2,08 \\
\hline $20-49$ & $29,27 \%$ & $7,32 \%$ & 2,07 \\
\hline $50-99$ & $42,31 \%$ & $15,38 \%$ & 2,38 \\
\hline $100-199$ & $66,67 \%$ & $50,00 \%$ & 3,17 \\
\hline$>200$ & $25,00 \%$ & $0,00 \%$ & 2,50 \\
\hline Total & $\mathbf{3 8 , 0 6} \%$ & $\mathbf{1 4 , 1 8} \%$ & 2,37 \\
\hline
\end{tabular}

* Nota: Valores medios entre 1 (nada importante) y 5 (muy importante).

Fuente: Elaboración propia mediante encuesta a 134 empresas, realizada en 2008. 
Del mismo modo, el grado de utilización de sus servicios resulta muy bajo, especialmente porque tras la integración de la EPSA en la UPV y la posterior creación del Instituto Tecnológico Textil (AITEX), muchos de los servicios prestados a las empresas en los laboratorios textiles pasaron a prestarse por esta última institución. No obstante, sí que existe una cierta interrelación entre ciertos grupos de investigación de la EPSA y la industria, especialmente orientados más a la prestación de servicios que a la investigación, si bien, esta orientación se está perdiendo tras la reforma de la Ley de Universidades, que concede mayor importancia al currículo investigador del personal universitario. De hecho, fruto probablemente de esta evolución, la importancia que la industria concede al papel desempeñado por la Universidad se encuentra en valores medios más próximos a nada importante (2) que a importante.

\section{b) Instituto Tecnológico Textil}

AITEX, fundado en el año 1985 por iniciativa del gobierno regional valenciano, mantiene una posición crucial como centro de referencia para la investigación, innovación y prestación de servicios avanzados para la industria textil-confección. Cabe resaltar que durante la época de su creación la oferta de investigación de la universidad era mínima y no tenía un órgano de gestión como es el actual Centro de Transferencia de Tecnología, por ello, AITEX se configuró como el primer centro de investigación en el sector textil, acaparando la mayoria de los servicios que se prestaban en los laboratorios universitarios. Constituida como asociación sin ánimo de lucro, el Centro promueve la modernización e introducción de tecnologias nuevas y emergentes a través de proyectos de I+D y, en general, a través de acciones que contribuyen al progreso industrial de sus socios. El número de empresas asociadas ha crecido continuadamente desde su creación, si bien, cualquier empresa puede aprovecharse de sus servicios, aunque no reúna la condición de socio (Gráfico 4).

Respecto a la distribución geográfica, se puede observar una tendencia que va desde la regionalización a la dispersión geográfica, ya que si en 2004 la proporción de empresas ajenas a la región representaban poco más del $50 \%$, en la actualidad este porcentaje se ha elevado hasta algo más del $60 \%$.

Teniendo en cuenta el tamaño de las empresas, se aprecia cómo las grandes empresas tienden a ser miembros de esta asociación, mientras que las empresas pequeñas consideran menos importante su aportación al negocio por

GRÁFICO 4. EVOLUCIÓN DEL NÚMERO DE EMPRESAS ASOCIADAS A AITEX (2000-2009)

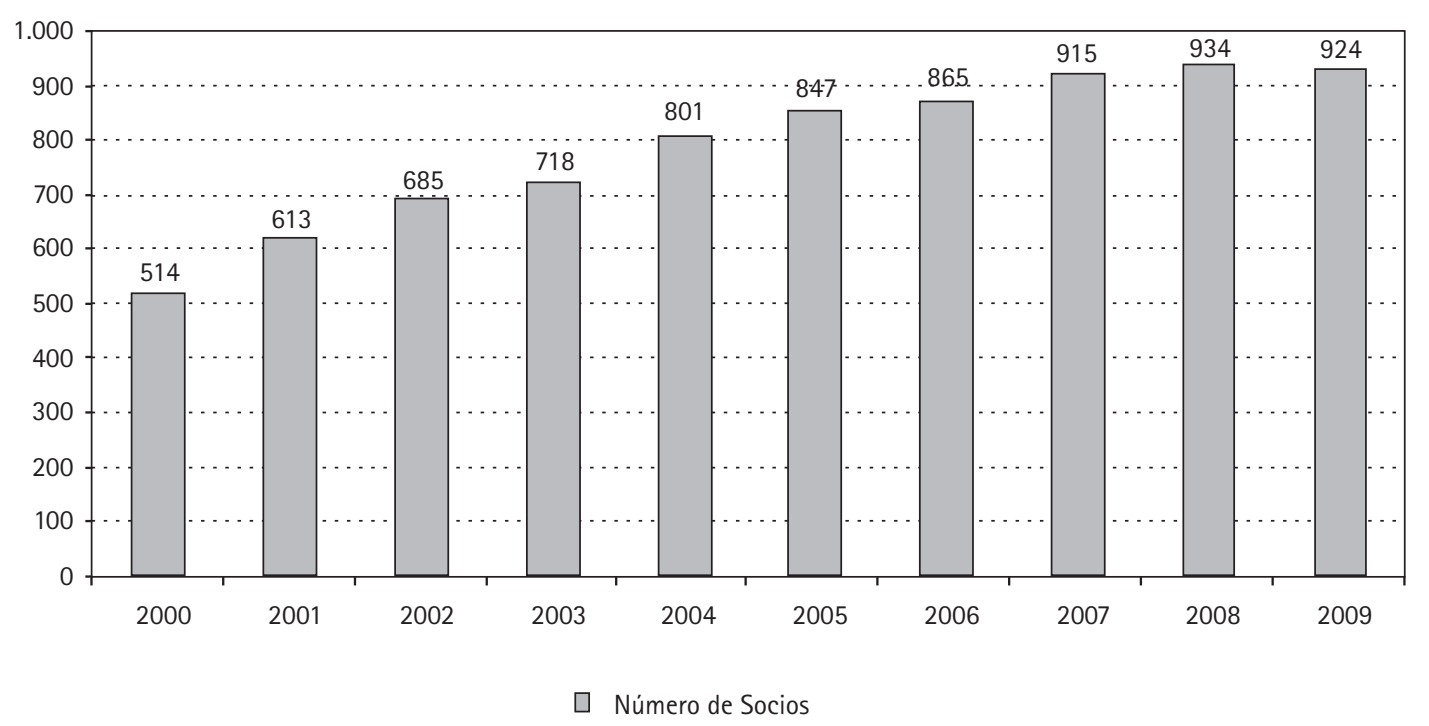

Fuente: AITEX (2010). 
parte de esta institución. Más del $80 \%$ de las empresas con más de 50 trabajadores son socios de AITEX y por el contrario, sólo el 32\% de las empresas con menos de 10 trabajadores lo son.

El número de servicios avanzados que AITEX provee a la industria es bastante elevado. Según un estudio específico sobre la percepción de los servicios provistos por AITEX la mayoria de las respuestas fueron favorables, con un promedio de 3,23 (importante) que crecía hasta el 3,63 entre los establecimientos que sí eran socios. Estos servicios son realmente importantes para conseguir niveles de calidad que son difíciles de imitar por productores localizados en países de bajo coste. Los servicios de laboratorio son los más demandados por la industria y son seguidos por las certificaciones de calidad que mejoran los requerimientos de los clientes.

\subsection{Agentes empresariales}

\section{a) ATEVAL: "Asociación de Empresarios Textiles de la Comunidad Valenciana"}

Esta asociación, formada por algo más de 500 empresas, es la principal representación del sector, proveyendo a sus socios de servicios como asesoramiento legal y comercial, así como cursos de formación específica. De la misma forma, ATEVAL coordina el programa "Textil Hogar de España" en el cual las empresas participantes muestran una oferta conjunta de sus productos vía Internet y vía Ferias. ATEVAL, como patronal, negocia los convenios colectivos para estipular, por ejemplo, las jornadas laborales, las vacaciones y los derechos de los trabajadores.

Actualmente, ATEVAL mantiene varios acuerdos con el resto de agentes del sistema de innovación. Por ejemplo, promueve las relaciones universidad-empresa a través de estancias cortas de estudiantes en empresas textiles. Mantiene una comunicación fluida con la oficina de prácticas de empresas y servicio de empleo de la EPSA. Adicionalmente, ATEVAL coordina la acción colectiva promoviendo los productos y servicios de las empresas textiles a través de las ferias internacionales (principalmente la de Frankfurt): gestionando las ayudas al comercio exterior, reservando los espacios en las mismas y contactando con posibles clientes. En los últimos años, ha aumentado espectacularmente sus servicios a las empresas (orientación estratégica, a través de la oficina ESTRATEX, gestión de subvenciones, etc.), dado que es la institución encargada de gestionar el Plan de Competitividad Textil, implantado por la Conselleria de Industria, tras la irrupción de la crisis provocada por la apertura de mercados en 2005.

\section{b) La Asociación Textil Alcoyana}

Se trata de la asociación local más antigua de industriales en la zona. Si bien fue pionera en defender los intereses de los industriales textiles alcoyanos, tras sufrir varias reformas a lo largo del siglo $X X$, actualmente se dedica a proveer servicios específicos a más de 100 empresas en materia de asesoría legal, medioambiental y administrativa. La gestión de subvenciones es el principal servicio que hoy en día ofrece a sus asociados.

Con respecto a sus relaciones con otros agentes del sistema de innovación, esta asociación participa en acuerdos de colaboración con AITEX y ATEVAL, las cuales le transfieren parte de su exceso de carga laboral, sobre todo en materia de gestión de nóminas, impuestos y tasas.

\section{LA DINÁMICA DEL SISTEMA DE INNOVACIÓN en la industria textIL de la CCV}

En el apartado anterior se han descrito cada uno de los agentes principales que conforman el sistema local de innovación en las Comarcas Centrales Textiles Valencianas. A continuación, se pretende ofrecer una aproximación acerca de cómo algunos de estos agentes han actuado como fuentes de innovación y de qué modo otros componentes han condicionado este proceso.

En primer lugar, cabe destacar que el principal subsistema es el industrial, pues son las empresas las que introducen las innovaciones en el mercado. Ahora bien, la articulación espacial de la industria como un distrito industrial, en el cual existe una amplia dispersión de empresas ubicadas en todas y cada una de las fases de la cadena de valor sectorial, conlleva que la posibilidad de subcontratar territorialmente parte de los procesos no integrados en la empresa genere un efecto tirón sobre todas estas actividades, desde las empresas ubicadas en las últimas fases de la cadena de valor. Estas empresas normalmente pertenecen al segmen-

ARBOR Vol. 188753 enero-febrero [2012] 75-96 ISSN: 0210-1963 
to de textil-hogar y poseen estrategias de diferenciación (imagen de marca, diseño, integración hacia la decoración, elevado grado de internacionalización, etc.) más acusadas. Al margen, lógicamente los sectores proveedores tanto de tecnología como de inputs ejercen un efecto empuje sobre el resto de la cadena de valor (acabadores, hilatura, fibras, etc.), que incorpora como elemento diferenciador las innovaciones desarrolladas en los mismos, si bien mantienen un bajo grado de apropiabilidad de las mismas. Esta última circunstancia genera que, dada la existencia de relaciones empresariales regidas por cuestiones ligadas a la proximidad espacial, más allá de la mera lógica económica, rápidamente se genere un efecto de imitación y la disrupción competitiva provocada por la introducción de innovaciones, quede absorbida por el conjunto de la cadena de valor sectorial.

No obstante, conviene indicar que uno de los fenómenos más claros que se observan a raíz de la creciente liberalización del mercado mundial de textil-confección es la creciente desarticulación de estas relaciones tradicionales, debido tanto a la creciente incorporación de producto importado por las empresas más diferenciadas, como a la necesidad de las ubicadas en segmentos de cabecera de completar su mercado mediante la integración hacia adelante, asumiendo funciones de sus tradicionales clientes. Desde este punto de vista, la tendencia en la dinámica espacial de la innovación claramente se ha visto alterada, generando un mayor nivel de ostracismo en las relaciones cliente-proveedor, tanto por la deslocalización productiva como por la tendencia hacia la integración de un número mayor de procesos por parte de las empresas más grandes, que a su vez ha reducido los beneficios derivados de la aglomeración local de la industria.

Por lo que respecta al papel ejercido en la dinámica de innovación sectorial por parte de las instituciones de educación e investigación, cabe indicar que en el caso del sistema universitario de manera progresiva se ha producido un desplazamiento hacia la investigación básica y el desarrollo de currículos formativos no tradicionales en la zona. En el primer caso, la integración de la EPSA en la UPV en 1972 y la introducción de criterios de evaluación estándar del personal docente e investigador a nivel nacional, provocó tanto una progresiva pérdida de interés curricular del personal universitario en la prestación de servicios a la industria, como una barrera de entrada, casi inapreciable hasta la fecha, que acotaba la movilidad entre la universidad y la industria, y viceversa. Bajo este prisma, el salto cualitativo de la universidad conlleva una pérdida de contacto con la industria local ante la baja capacidad de absorción de la industria, tanto en términos de conocimiento como en términos de personal universitario con un nivel de cualificación excesivamente alto para sus necesidades. Obviamente, aspectos como la creación del Área de Relaciones con el Entorno en la EPSA han incentivado el papel de estructura de interfaz de la misma en el sistema de innovación local, no obstante, frente a la clara especialización sectorial existente en el sistema anterior, construido de forma tácita desde la creación de la Escuela, esta nueva dimensión ha resultado completamente trasversal, llevando a una pérdida paulatina de relevancia en la dinámica de innovación del sector.

En este sentido, cabe incidir en la centralidad en el sistema de innovación adquirida por el Instituto Tecnológico Textil, que en un momento en el que la normalización de producto resultaba crucial para garantizar el acceso al mercado europeo, pasó a ocupar el papel que tradicionalmente había realizado la EPSA, como laboratorio técnico, pero con capacidad de certificación y unos medios técnicos superiores. La propia forma jurídica de AITEX, como asociación de fabricantes, lo posicionó como un elemento clave de la política industrial valenciana orientada al sector, favoreciendo su crecimiento y su posterior multilocalización, ante la caída drástica de la actividad en la industria local. De hecho, si bien en los últimos años éste ha tratado de focalizar gran parte de su oferta tecnológica y de investigación hacia el segmento de textiles técnicos, tanto la falta de industria tractora que ejerciera una demanda en cantidad tanto a nivel local, como regional y nacional, como la falta de capacitación técnica de la industria para enfrentarse a segmentos más intensivos en conocimiento, ha llevado a la necesidad de ampliar su radio de acción fuera de su ámbito original de creación.

Por lo que respecta a las asociaciones empresariales, ya desde los años setenta se produjo una lenta transformación por la cual, frente a la típica defensa de la burguesía industrial local, se fueron introduciendo elementos de profesionalización de los servicios prestados a la industria, especialmente en áreas como la internacionalización y la formación continua. En los últimos años, su papel como estructura de interfaz en el sistema de innovación sectorial 
claramente se ha visto reforzado por la centralidad adquirida en las medidas que desde el Gobierno valenciano se han impulsado para tratar de paliar la situación de crisis sectorial.

Por último, las instituciones financieras si bien han impulsado y apoyado tradicionalmente el despegue de muchas empresas locales, debido a la cercanía y al trato personal, su deriva hacia el sector de la construcción, y su pérdida de poder de toma de decisión (al depender de órganos más elevados, por ejemplo, las centrales de riesgos de las propias entidades) han generado un desequilibrio entre las necesidades financieras de las empresas y su cobertura.

\section{Conclusiones}

La estructura económica en la Comunidad Valenciana, así como en otras regiones, está cambiando dramáticamente en los últimos años. El proceso de globalización está forzando a llevar a cabo una serie de cambios en la mayoría de industrias tradicionales, incluyendo la actividad textilconfección. En este contexto, la clase política, empresarios y la sociedad en su conjunto tiene que repensar cómo recobrar competitividad pero basada en la innovación, en lugar de apoyarse en estrategias basadas en la reducción de costes.

La aglomeración de industrias textiles en un distrito industrial específico alrededor de una serie de municipios como Alcoi, Ontinyent y Bocairent, ha asegurado históricamente una buena provisión de servicios de apoyo por parte de diferentes instituciones localizadas en este ámbito geográfico. Sin embargo, el sector está atravesando una situación de reestructuración y pérdida de peso importante durante los últimos años. Desde un punto de vista sistémico, primeramente, la carencia de financiación de las empresas por parte de las entidades financieras ha obstaculizado la inversión en la modernización del sector textil. No obstante, también es destacable la tendencia por parte de empresarios a invertir en otros sectores con unos mayores beneficios a corto plazo, que han tenido como consecuencia más grave el cierre de muchas empresas textiles. Obviamente, ambas razones han generado unos efectos negativos en el entorno socioeconómico y sobre todo una visión pesimista hacia el sector por parte de los jóvenes, que no suelen incluir a las empresas textiles en su lista de preferencias laborales.

Por otro lado, la concentración de los canales de distribución en unas pocas grandes empresas es otro factor que explica la falta de competitividad en el sector textil valenciano. Actualmente, los grandes distribuidores y las cadenas de tiendas especializadas no sólo controlan los principales mercados, sino que han provocado una progresiva desaparición de la base de demanda de la industria local y nacional: el comercio minorista. La gran concentración de la oferta en estos grupos de distribución, que se proveen normalmente de productos provenientes de empresas localizadas en paises de bajo coste, ha reducido igualmente la demanda de productos y procesos intermedios de muchas empresas locales.

En este contexto, parece que frente a las cuestiones productivas ligadas a la aglomeración en un territorio concreto de un gran número de productores de procesos y productos finales, la relevancia actual del sistema de demanda dentro del esquema del sistema de innovación parece crucial. Esta configuración, basada en el control de la distribución, permite un mejor entendimiento de las tendencias de moda y de la relación empresa-cliente, que ha sido una de las claves del éxito comercial, junto con la gestión logística, de las empresas mejor posicionadas.

Precisamente esta falta de posicionamiento en el mercado explica el interés de las industrias textiles locales por la diversificación al textil técnico, ya que los canales de distribución en este nicho son más reducidos que los tradicionales y por lo tanto la competencia es menos agresiva. La diversificación hacia este segmento permite un aprovechamiento del conocimiento textil, que normalmente comporta un mayor valor añadido en el sector de aplicación, dado su carácter técnico. En el caso de las CCV, el hecho de contar con instituciones tales como AITEX y la EPSA pueden ayudar enormemente a transformar una industria tradicional en una industria de vanguardia. No obstante, una limitación observada en este proceso de transición o diversificación es la carencia de proveedores especializados (principalmente de fibras y productos químicos y maquinaria especializada), así como la carencia de personal con conocimientos no sólo textiles, sino también del sector de aplicación y, de forma determinante, la ausencia de industrias deman- 
dantes de este tipo de productos. Otra está relacionada con la tendencia a interiorizar el proceso de innovación de este tipo de productos, dada su fácil replicabilidad, tanto por empresas, como por las instituciones de carácter tecnológico, que actúan como prescriptores y difusoras de conocimiento. De hecho, en la actualidad existen pocos ejemplos de empresas que cooperen con agentes de investigación y tecnológicos en este mercado, dado que, en un sector dependiente de las innovaciones químicas y de maquinaria, el secretismo es uno de los pocos mecanismos de apropiación de innovaciones.

Es también destacable que más allá de solicitar la provisión de servicios avanzados, pocas empresas reconocen trabajar con el personal universitario en proyectos de investigación conjuntos. En este sentido, la universidad cabría repensar y adecuar su papel en este tipo de acciones. Por ejemplo, actualmente la mayoría de contratos de la universidad con la industria se basan en la provisión de servicios avanzados que ya son ofertados en el mercado, ya sea a través de empresas de consultoría como por la red de institutos tecnológicos. En su lugar, una mayor orientación hacia la investigación básica o aplicada eliminaría esta competencia directa entre agentes localizados en el mismo ámbito geográfico.

Finalmente, con respecto a la imagen negativa de la industria textil, generada principalmente por los medios de comunicación, todas las instituciones deberían realizar un mayor esfuerzo para incentivar a los jóvenes, por una parte, y a los actuales empresarios, por otra, a lograr llevar a buen puerto este proceso de cambio. De esta manera, los jóvenes aprenderian sobre su herencia industrial y mejorarian la situación actual protagonizada por un nuevo escenario, por ejemplo, a través de concursos de diseño textil, exposiciones de colecciones de moda u ofertas de trabajo y prácticas en empresas. De hecho, echando la vista atrás desde el siglo XII esta actividad en esta área se ha caracterizado por su habilidad para adaptarse a nuevos desafíos y entornos turbulentos.
NOTA

1 Para más información sobre la historia de esta institución, consultar los siguientes trabajos: Blanes-Nadal (1999); Blanes-Nadal et al. (2000) y Garrigós-Oltra y Blanes-Nadal (2001). Actualmente, se denomina Campos de Alcoy (Universidad Politécnica de Valencia).

\section{BIBLIOGRAFÍA}

Acotex (2009): "El comercio en cifras", Madrid, Informe anual de la Asociación de empresarial del comercio textil y complementos.

Aitex (2010): Información sobre AITEX [en línea] [ref. de 5 de septiembre 2010] Disponible en web: http://www.aitex. es/index.php? option=com_content\&t ask=view\&id $=309 \&$ Itemid $=384$
Alto Consejo Consultivo (2007): La innovación en el sector textil-confección de la Comunidad Valenciana, Alto Consejo Consultivo de la Comunidad Valenciana, IMPIVA, p. 209.

Arnold, E. y Kuhlmann, S. (2001): RCN in the Norwegian Research and Innovation System, informe de base n. ${ }^{\circ} 12$ de la evaluación del Consejo de Investigación de Noruega, Oslo: Ministerio de Educación, Investigación y Asuntos Religiosos del Reino de Noruega [ref. de 1 de diciembre 2000]. Disponible en web: www.technopolis-group.com

Barge-Gil, A. y Modrego-Rico, A. (2007): "Los centros tecnológicos como instrumentos de intervención pública en los sistemas regionales de innovación", en Vence Deza, X. (coord.), Crecimiento y políticas de innovación. Nuevas tendencias y experiencias comparadas, Madrid: Ed. Pirámide, pp. 241-272.
Aceptado: 15 de junio de 2011 
Blanes Nadal, G. (1999): Educación e innovación tecnológica en el proceso de industrialización alcoyana, Dpto. Ingeniería Textil y Papelera, EPS de Alcoy, UPV. Tesis doctoral inédita.

Blanes Nadal, G.; Garrigós Oltra, L.; Millán Verdú, C. y Sebastiá Alcaraz, R. (2000): Orígenes de la enseñanza técnica en Alcoy, Instituto Alicantino de Cultura Juan Gil Albert, Quinta Impresión S.L.

Bonaccorsi, A. y Piccaluga, A. (1994): "A Theoretical Framework for the Evaluation of University-Industry Relationships" en R\&D Management vol. 24, n. 3 , pp. 229-247.

Breschi, S. y Malerba, F. (1997): "Sectoral Innovation Systems: Technological Regimes, Schumpeterian Dynamics, and Spatial Boundaries", en C. Edquist (ed.): Systems of Innovation: Technologies, Institutions and Organizations, cap. 6, Londres y Washington: Pinter, pp. 130-156.

Cambra-Colmenero, J. L. y Golf-Laville, E. J. (2007): Empresa Extendida: Análisis de estrategias de diversificación e integración en la Industria Textil Valenciana, Proyecto Final de Carrera. Escuela Politécnica Superior de Alcoy. Universidad Politécnica de Valencia, p. 117.

Capó-Vicedo, J.; Expósito-Langa, M. y Molina-Morales, X. (2008): "Improving SME competitiveness reinforcing interorganisational networks in industrial clusters", en The International Entrepreneurial and Management Journal, 4 (2), pp. 147-169.

Carlsson, B.; Jacobsson, S.; Holmén, M. y Rickne, A. (2002): "Innovation Systems: Analytical and Methodological Issues", en Research Policy, n. ${ }^{\circ} 31$, pp. 233-245.

Castellaci F.; Grodal, S.; Mendonca, S. y Wibe, M (2005): "Advances and challenges in Innovation Studies", en Journal of Economic Issues, Vol. XXXIX n. ${ }^{\circ}$, pp. 91-121.
Cohen, W. M. y Levinthal, D. (1990): "Absortive Capacity: a new Perspective on Learning and Innovation", en Administrative Science Quarterly, n. 35 (1), pp. 128-192.

De la Mothe, J. y Paquet, G. (1998): Local and regional systems of innovation, Massachusetts: Kluwer Academic Publishers, p. 370.

Dei Ottati, G. (2005), "Global competition and entrepreneurial behaviour in industrial districts: trust relations in an Italian industrial district", en $\mathrm{H}$. Höhmann y F. Welter (Eds.), Trust and Entrepreneurship: A West-East Perspective, Cheltenham: Edward Elgar Publishing, pp. 255-271.

Dosi, G.; Freeman, C.; Nelson, R.; Silverberg, G. y Soete, L. (1988): Technical Change and Economic Theory, London: Pinter, p. 656.

Fagerberg, J. y Verspagen, B. (2009): "Innovation studies-The emerging structure of a new scientific field", en Research Policy, Vol. 38, n. ${ }^{\circ}$ 2, pp. 218-233.

Fernández de Lucio, I.; Conesa, F.; Garea, M.; Castro, E.; Gutiérrez, A. y Bodegas, M. A. (1996): Estructuras de interfaz en el Sistema español de Innovación. Su papel en la difusión de tecnología, Centro de Transferencia de Tecnología, Valencia: Servicio de Publicaciones de la Universidad Politécnica de Valencia.

Freeman, C. (1982): "Technological Infrastructure and International Competitiveness", en Industrial and Corporate Change, Vol. 13, n. 3 [2004]: pp. 541 569.

Gabinete Sigma (1974): La economía de Alcoy y comarca: aspectos históricos y estructuras actuales, Valencia: Monte de Piedad y Caja de Ahorros de Alcoy, p. 400.

Galanakis, K. (2006): "Innovation process. Make sense using systems thinking", en Technovation, n. ${ }^{\circ} 26$, pp. 12221232.
García Aracil, A. y Fernández de Lucio, I. (2008): "Industry-University Interactions in a Peripheral European Region: An Empirical Study of Valencian Firms", en Regional Studies, Vol. 42, n. ${ }^{\circ}$, pp. 215-227.

Garrigós Oltra, L. y Blanes Nadal, G. (2001): 150 anys de la consolidació de l'ensenyament industrial a Alcoi, Alicante: Quinta Impresión S.L.

Godin, B. (2007): "National Innovation Systems: The System Approach in Historical Perspective", Working Paper n. ${ }^{\circ} 36$, Project on the History and Sociology of STI Statistics. Canadá.

Golf Laville, E. J.; Molina, M. J.; Pla Barber, J. y Puig Blanco, F. (2005): Plan Estratégico Textil de las CCV, Ayuntamiento de Ontinyent, p. 289.

Golf Laville, E. J. (2007): Canales de Distribución de Textiles Técnicos, Alcoy: UPV, p. 182.

Golf Laville, E.; Pla Barber, J. y Molina, M. J. (2008): Sistemas de innovación de textiles técnicos en la Unión Europea. Alcoy: ATEVAL, p. 253.

INE (2010): Directorio Central de Empresas, Instituto Nacional de Estadística, Madrid.

Kitagawa, F. (2005): "Entrepreneurial Universities and the Development of regional societies: a Special View of the Europe of Knowledge", en Higher Education Management and Policy, Vol. 17, n. ${ }^{\circ}$, OECD, pp. 65-90.

Lester, R. K. (2005): "Universities, Innovation and Local Economies". A Summary Report from the Local Innovation Systems Project - Phase 1. MIT Industrial Performance Center Working Paper 05-010 [ref. de 10 de agosto 2004]. Disponible en web: http://web.mit. edu/lis/papers/LIS05-010.pdf

Lundvall, B. A. (1992): National Systems of Innovation: Towards a Theory of Innovation and Interactive Learning, London: Pinter, p. 342. 
Malerba. F. (2002): "Sectoral systems of innovation and production", en Research Policy, Vol. 31, n. 2, pp. 247-264.

Marshall, C. y Rossman, G. B. (1998). Designing Qualitative Research (3rd ed.), Sage Publications, Inc.

Moulaert, F. y Hamdouch, A. (2006): "New views of Innovation Systems. Agents, rationales, networks and spatial scales in the knowledge infrastructure", en Innovation, Vol. 19, n. ${ }^{\circ} 1$, pp. 11-24.

Mytelka, L. (2000): "Local Systems of Innovation in a Globalized World Economy", en Industry and Innovation, Vol. 7, n. ${ }^{\circ}$ 1, pp. 15-32.

Nelson, R. R. (1993): National Innovation Systems: A comparative Analysis, 0xford: Oxford University Press, p. 560.

OCDE (2001): Examen de la OCDE sobre las Comarcas Centrales de la Comunidad Valenciana, Universidad Politécnica de Valencia, p. 177.

Pavitt, K. (1984): "Sectoral Patterns of Technical Change: Towards a Taxo- nomy and a Theory", en Research Policy, Vol. 13, pp. 343-373.

Pinto, H. (2009): "The Diversity of Innovation in the European Union: Mapping Latent Dimensions and Regional Profiles" en European Planning Studies, Vol. 17, n. 2 , pp. 303-326.

Robertson, P. L. y Smith, K. (2009): "Distributed knowledge bases in low- and medium technology industries", en Hirsch-Kreinsen, H. y Jacobson, D. (eds.): Innovation in Low- and Medium-Tech Firms and Industries, Cheltenham: Edward Elgar, pp. 93-118.

SABI (2010): Sistema de Análisis de Balances lbéricos, Base de Datos en CDROM. Acceso desde la Universidad Politécnica de Valencia.

Santamaría, L.; Nieto, M. J. y Barge-Gil, A. (2009): "Beyond the formal R\&D: Taking advantage of other sources of innovation in low- and mediumtechnology industries", en Research Policy, Vol. 38, pp. 507-517.
Shattock, M. (2005): "European Universities for Entrepreneurship: Their Role in the Europe of Knowledge. The Theoretical Context", en Higher Education Management and Policy, Vol. 17, n. ${ }^{\circ} 3$, OECD, pp. 13-27.

Storey, D. J. y Tether, B. S. (1998): "Public Policy measures to support new technology based firms in the European Union" en Research Policy, Vol. 26, Issue 9, pp. 1037-1057.

Such, J. (1993): La industria textil de AlcoiOntinyent: Problemas y perspectivas, Caja de Ahorros del Mediterráneo, Alcoi, Alicante.

Vallés, I. (1986): Indústria Textil I Societat a la Regió Alcoi-Ontinyent 1780-1930, Institut Valencià d'Estudis i Investigació, Universitat de València, p. 197.

Ybarra, J. A. (1991): "Determinación cuantitativa de distritos industriales: la experiencia del País Valenciano", en Estudios Territoriales, n. ${ }^{3}$ 37, pp. 5367. 